\title{
Tracking a Maneuvering Target using AUV
}

\author{
P. Dhatri Shree, M. Ajay Kumar, M. Sai Charan, S. Koteswara Rao, Kausar Jahan
}

\begin{abstract}
In this paper effort is made to track a maneuvering target using Unmanned Aerial Vehicles (UAV) with range, bearing and elevation measurements. Extended Kalman filter is preferred to processmeasurements tampered with noise. Algorithm to detect the maneuver of target is developed in this paper. This information about range, bearing and elevation is communicated to weapon guidance station by means of personal communication system between UAV and weapon guidance station. Mathematical modeling in detail and simulation results is presented.
\end{abstract}

Keywords: Estimation, Extended Kalman Filter, Maneuvering Target Motion Analysis, Three-Dimensional tracking, Unmanned aerial vehicle.

\section{INTRODUCTION}

This aerial vehicle (UAV) is the safest airborne warfare system existing in the world today. UAV is a robot-like system flying in air mainly used in target tracking. It sends radio waves to track the target parameters like range, bearing and elevation. UAV now-a-days are GPS equipped so that weapon guidance system of UAV will have knowledge about position of UAV. Weapon guidance system may be a ship on the surface or an aircraft in air. Data received from UAV is sent to weapon guidance system by means of a personal communication system so that weapon guidance system will be able to know the position and motion of target and releases weapon in that direction. Target tracking is carried out using Extended Kalman filter (EKF). Target motion parameters particularly at long ranges are nonlinear. So, EKF is considered based on rapidly convergent and unbiased filter problems in Kalman Filter [9-17].

Tracking of the target is carried out by Extended Kalman filter (EKF) [3-9]. In this paper, the main contribution is

Revised Manuscript Received on December 30, 2019.

* Correspondence Author

P. Dhatri Shree, B. Tech Student, Department of Electronics and Communication Engineering, Koneru Lakshmaiah Education Foundation, Vaddeswaram, Guntur, A.P, India.

M. Ajay Kumar, B. Tech Student, Department of Electronics and Communication Engineering, Koneru Lakshmaiah Education Foundation, Vaddeswaram, Guntur, A.P, India.

M. Sai Charan, B. Tech Student, Department of Electronics and Communication Engineering, Koneru Lakshmaiah Education Foundation, Vaddeswaram, Guntur, A.P, India.

S. Koteswara Rao, Professor, Department of Electronics and Communication Engineering, Koneru Lakshmaiah Education Foundation, Vaddeswaram, Guntur, A.P, India.

*Kausar Jahan, Research Scholar, Department of Electronics and Communication Engineering, Koneru Lakshmaiah Education Foundation, Vaddeswaram, Guntur, A.P, India. Corresponding

(C) The Authors. Published by Blue Eyes Intelligence Engineering and Sciences Publication (BEIESP). This is an open access article under the CC BY-NC-ND license (http://creativecommons.org/licenses/by-nc-nd/4.0/) tracking of a maneuvering target, as suggested in [4, 5] Target maneuver cannot be visualized easily by observing bearing residual plot. So, zero mean chi-square distributed random sequence residuals in sliding window is used for detecting maneuver of the target. Normalized squared innovation process is used to find out whether target is maneuvering or not. To get the finest solution during target maneuver enough amount of process noise is added to the covariance. When the maneuver is completed, state noise is lowered back. Bearing and elevation measurements are non-linearly related to the state of the target parameters making the process non-linear. So, the optimal linear filters like Kalman filter is not suitable for three-dimensional tracking of target. For simplicity of the process, the target is assumed to be travelling with constant speed and maneuvers in its course. The plant noise considered is white Gaussian noise generated due to disturbance in the velocity of target.

Section II contains with mathematical modeling and implementation of the process. Section II also deals with generation of measurements in simulation environment. Sections III describe simulation and the results obtained. The paper is concluded in Section IV.

\section{MATHEMATICAL MODELING}

\section{A. General Simulator model}

Author Let initial position of the target and observer be $\left(x_{t}, y_{t}, z_{t}\right)$ and $\left(x_{0}, y_{0}, z_{0}\right)$, assuming that they move with velocities $v_{t}$ and $v_{0}$. After $t$ seconds, change in observer position is given as follows.

$$
\begin{aligned}
& d x_{0}=v_{0} \sin (o c r) \sin (o p h) t \\
& d y_{0}=v_{0} \cos (o c r) \sin (o p h) t \\
& d z_{0}=v_{0} \cos (o p h) t
\end{aligned}
$$

Here oph and ocr are pitch and course of the observer respectively. The changed observer position is given as follows.

$$
\begin{aligned}
& x_{0}=x_{0}+d x_{0} \\
& y_{0}=y_{0}+d y_{0} \\
& z_{0}=z_{0}+d z_{0}
\end{aligned}
$$

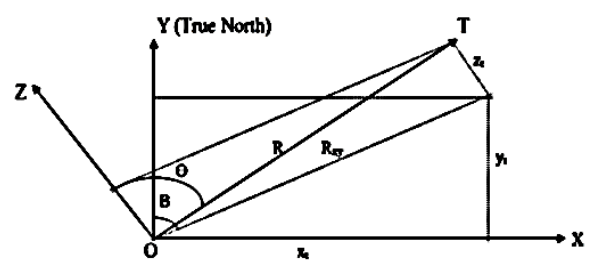

Fig.1.Target and observer positions 
Similarly, from Fig.1.

$x_{t}=R_{x y} \sin (B)$

$y_{t}=R_{x y} \cos (B)$

$\sin (\theta)=R_{x y} / R$

Substituting (35) in (33) and (34),

$x_{t}=R \sin (\theta) \sin (B)$

$$
y_{t}=R \sin (\theta) \cos (B)
$$

$$
z_{t}=R \cos (\theta)
$$

Fig.2.Target and observer velocities

When the target is in motion with velocity $v_{t}$, change in target position after $t$ seconds, from Fig.2, is given as follows.

$$
\begin{aligned}
& d x_{t}=v_{t} \sin (t c r) \sin (t p h) t \\
& d y_{t}=v_{t} \cos (t c r) \sin (t p h) t \\
& d z_{t}=v_{t} \cos (t p h) t
\end{aligned}
$$

Here $t p h$ and $t c r$ are pitch and course of the target respectively.

The changed target position is as follows.

$$
\begin{aligned}
& x_{t}=x_{t}+d x_{t} \\
& y_{t}=y_{t}+d y_{t}
\end{aligned}
$$

$z_{t}=z_{t}+d z_{t}$

Simulated true values of bearing, range and elevation of the target are given as follows.

$$
\begin{aligned}
& \text { true bearing }=\tan ^{-1}\left(\left(x_{t}-x_{0}\right) /\left(y_{t}-y_{0}\right)\right) \\
& \text { true range }=\sqrt{\left(x_{t}-x_{0}\right)^{2}+\left(y_{t}-y_{0}\right)^{2}+\left(z_{t}-z_{0}\right)^{2}} \\
& \text { true elevation }=\tan ^{-1}\left(R_{x y} / z_{t}-z_{0}\right)
\end{aligned}
$$

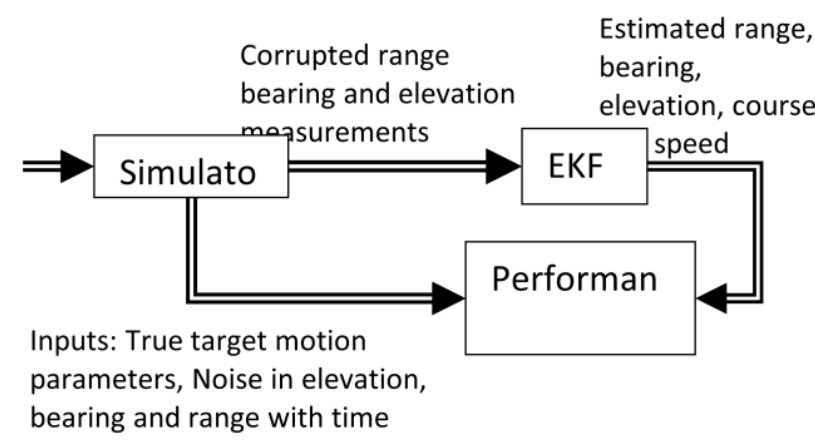

Fig:3. Block diagram of TMA in simulation mode

Block diagram of target motion analysis in simulation mode is shown in Fig.3. The measurements tampered with noise are utilized for the prediction of target parameters using EKF. The estimated target parameters are compared with that of true values.

\section{B. System model}

Consider state vector

$$
X_{S}(k t)=\left[\begin{array}{c}
\dot{x}(k t) \\
\dot{y}(k t) \\
\dot{z}(k t) \\
R_{x}(k t) \\
R_{y}(k t) \\
R_{z}(k t)
\end{array}\right]
$$

Here $\dot{x}(k t), \dot{y}(k t), \dot{z}(k t)$ are speed parameters of the target and $R_{x}(k t), R_{y}(k t), R_{z}(k t)$ are its range parameters in $x, y$ and $z$ directions respectively. The state equation becomes

$$
X_{s}(k t+1)=\emptyset X_{s}(k t)+b(k t+1)+\Gamma w(k t)
$$

$\emptyset$ is given by

$$
\varnothing=\left[\begin{array}{llllll}
1 & 0 & 0 & 0 & 0 & 0 \\
0 & 1 & 0 & 0 & 0 & 0 \\
0 & 0 & 1 & 0 & 0 & 0 \\
t & 0 & 0 & 1 & 0 & 0 \\
0 & t & 0 & 0 & 1 & 0 \\
0 & 0 & t & 0 & 0 & 1
\end{array}\right]
$$

Here $t$ is time interval at which measurement is obtained. $b(k t+1)$ is deterministic control matrix and is given by

$$
b(k t+1)=\left[\begin{array}{c}
0 \\
0 \\
0 \\
-\left(x_{0}(k t+1)+x_{0}(k t)\right) \\
-\left(y_{0}(k t+1)+y_{0}(k t)\right) \\
-\left(z_{0}(k t+1)+z_{0}(k t)\right)
\end{array}\right]^{T}
$$

Here $x_{0}, y_{0}, z_{0}$ are the observer position components. To reduce the mathematical complexity, $\mathrm{Y}$-axis is taken as reference while measuring all the angles. Let $w(k t)$ be plant noise

$$
\begin{aligned}
& w(k t)=\left[\begin{array}{lll}
w_{x} & w_{y} & w_{z}
\end{array}\right]^{T} \\
& \text { Variance of } w(k t) \text { is given by } \\
& E\left[\Gamma(k t) w(k t) w^{T}(k t) \Gamma^{T}(k t)\right]=Q \delta_{i j} \\
& \text { Where } \delta_{i j}=\sigma_{w}^{2}(i=k t)
\end{aligned}
$$

$=0$ otherwise

$$
\begin{gathered}
Q=\left[\begin{array}{cccccc}
t s^{2} & 0 & 0 & t s^{3} / 2 & 0 & 0 \\
0 & t s^{2} & 0 & 0 & t s^{3} / 2 & 0 \\
0 & 0 & t s^{2} & 0 & 0 & t s^{3} / 2 \\
t s^{3} / 2 & 0 & 0 & t s^{3} / 4 & 0 & 0 \\
0 & t s^{2} / 2 & 0 & 0 & t s^{3} / 4 & 0 \\
0 & 0 & t s^{2} / 2 & 0 & 0 & t s^{3} / 4
\end{array}\right] \\
\Gamma(k t)=\left[\begin{array}{ccc}
t & 0 & 0 \\
0 & t & 0 \\
0 & 0 & t \\
t^{2} / 2 & 0 & 0 \\
0 & t^{2} / 2 & 0 \\
0 & 0 & t^{2} / 2
\end{array}\right]
\end{gathered}
$$

$Z(k t)$ represents the matrix of all measurements and is given by

$$
Z(k t)=\left[\begin{array}{lll}
R_{m}(k t) & B_{m}(k t) & \Theta_{m}(k t)
\end{array}\right]^{T}
$$

Here $R_{m}(k t), B_{m}(k t)$ and $\theta_{m}(k t)$ are measured range, bearing and elevation

$$
\begin{aligned}
& R_{m}(k t)=R(k t)+\xi_{R}(k t) \\
& B_{m}(k t)=B(k t)+\xi_{B}(k t) \\
& \theta_{m}(k t)=\theta(k t)+\xi_{\theta}(k t)
\end{aligned}
$$

where $R(k t), B(k t)$ and $\theta(k t)$ are simulated range, simulated bearing and simulated elevation.

$$
\begin{aligned}
& R(k t)=\sqrt{R_{x}^{2}(k t)+R_{y}^{2}(k t)+R_{z}^{2}(k t)} \\
& B(k t)=\tan ^{-1}\left(R_{x}(k t) / R_{y}(k t)\right) \\
& \Theta(k t)=\tan ^{-1}\left(R_{x y}(k t) / R_{z}(k t)\right) \\
& \text { Where } R_{x y}=\sqrt{R_{x}^{2}+R_{y}^{2}} \\
& \text { Measurement vector is given by } \\
& Z(k t)=H(k t) X_{s}(k t)+\xi(k t)
\end{aligned}
$$




$$
\begin{aligned}
& H(k t) \\
& =\left[\begin{array}{cccccc}
0 & 0 & 0 & \sin (B) \sin (\theta) & \sin (\theta) \cos (B) & \cos (\theta) \\
0 & 0 & 0 & \frac{\cos (B)}{R_{x y}} & \frac{-\sin (B)}{R_{x y}} & 0 \\
0 & 0 & 0 & \frac{\sin (B) \cos (\theta)}{R} & \frac{\cos (\theta) \cos (B)}{R} & \frac{-\sin (\theta)}{R}
\end{array}\right]
\end{aligned}
$$

$$
\text { And } \xi(k t)=\left[\begin{array}{lll}
\xi_{R} & \xi_{B} & \xi_{\Theta}
\end{array}\right]^{T}
$$

\section{EKF Algorithm}

All EKF implementation is as follows.

i). Initially the estimation of state and its covariance be $X(0 \mid 0)$ and $P(0 \mid 0)$.

ii). State vector at the next time period is predicted as $X_{s}(k t+1)$ :

$X_{s}(k t+1)=\emptyset(k t+1 \mid k t) X_{k t}(k t)+b(k t+1)+$ $\omega(k t)$

iii). The predicted covariance matrix of the state vector is given as follows.

$P(k t+1 \mid k t)=\emptyset(k t+1 \mid k t) P(k t) \emptyset^{T}(k t+1 \mid k t)+$ $Q(k t+1)$

iv). The gain of the Kalman filter is calculated as given in eq. (44).

$G(k t+1)=P(k t+1 \mid k t) \emptyset^{T}(k t+1 \mid k t)[H(k t+$

1) $\left.P(k t+1 \mid k t) H^{T}(k t+1)+R\right]^{-1}$

v). The state estimation and its error covariance:

$X_{s}\left(k t+1 \mid(k t+1)=X_{s}(k t+1 \mid k t)+G(k t+\right.$

1) $[Z(k t+1)-\hat{Z}(k t+1)]$

$P(k t+1 \mid k t+1)=[1-G(k t+1) H(k t+$

1) $P(k t+1 \mid k t)]$

vi). For next iteration

$$
\begin{aligned}
& X_{s}(k t \mid k t)=X(k t+1 \mid k t+1) \\
& P(k t \mid k t)=P(k t+1 \mid k t+1)
\end{aligned}
$$

\section{Target Maneuver Detection}

When target is not maneuvering, the process noise is less. When target maneuvers, the process noise increases $[10,11]$. So, in simulation, the covariance matrix is multiplied by fledge factor of 10 for the time period of target maneuver. Once the target achieves its new course, i.e., completes the maneuver, process noise will be reduced. The normalized squared innovation, $\gamma_{\varphi}(k t)$, is calculated as follows.

$$
\begin{aligned}
& \gamma_{\varphi}(k t)=\varphi^{T}(k t) S^{-1}(k t+1) \varphi(k t+1) \\
& \text { Where } \varphi(k+1) \text { is } \\
& \varphi(k t+1)=Z(k t+1)-h(k t+1, X(k t+1 / k t)) \\
& \text { Let } S(k t) \text { is } \\
& S(k t+1)=H(k t+1) P(k t+1 / k t) H^{T}(k t+1)+\sigma^{2}
\end{aligned}
$$

Let

$d(\xi)=\gamma^{T} S^{-1} \gamma \geq c$

where $\mathrm{S}$ is $\operatorname{diag}\{S(k t)\}$

and $\gamma=\left[\begin{array}{lll}\varphi(1) & \varphi(2) \ldots \ldots \varphi(k t)\end{array}\right]^{T}$

where $c$ is a constant (threshold) and $d$ is chi-square distributed statistic. This sliding window size is chosen as 5 . evaluation of algorithm is shown in Table 2. For example, scenario1 describes a target at an initial range of $3000 \mathrm{~m}$ from the observer, travellingwith course and speeds of $170^{\circ}$ and $400 \mathrm{~m} / \mathrm{s}$ respectively. The initial line of sight is $0^{0}$. The bearing and range measurements are corrupted with 0.330 $(1 \sigma)$ and $10 \mathrm{~m}(1 \sigma)$ respectively. After 300s, target changes its course to $295^{\circ}$ at a turning rate of $3^{0}$ per second. The target elevation is $0^{0}$ for simplicity. The observer is assumed to travel with constant speed of $20 \mathrm{~m} / \mathrm{s}$ and with a course of $90^{\circ}$.

The measurements are assumed to be available continuously for every second. The true values of the target and observer are simulated in Matlab. So, the estimated values can be evaluated or validated based on the simulated values based on certain acceptance criteria.The acceptance criterion is chosen based on weapon control (this topic is not discussed here) requirement. The solutionis accepted or said to be converged when error in course estimate $<=3^{0}$ and error in speed estimate $<=1 \mathrm{~m} / \mathrm{s}$.

The estimates and true paths of target are shown in Fig.4 for scenario2.For clarity of the concepts, the simulated and predicted course and speed for scenario2 are presented in Fig.5and 6. Similarly simulated and predicted elevation of the target for scenario2 is presented in Fig.7.The solution is accepted or converged when the course and speed errors are within the acceptance criteria. The convergence time, in seconds, for the scenarios is given in Table.2.

The scenario2 chosen for evaluation of algorithm for maneuvering target has the convergence of solution for the estimated course of the target after maneuver at $460^{\text {th }}$ second and $67^{\text {th }}$ second before target maneuver respectively.The target does not maneuver in speed, so the convergence of speed estimate is 43 seconds for scenario 2.

Table. 1. Target to Observer Scenarios

\begin{tabular}{|l|c|c|}
\hline \multirow{2}{*}{ Parameters } & \multicolumn{2}{c|}{ Scenarios } \\
\cline { 2 - 3 } & $\mathbf{1}$ & $\mathbf{2}$ \\
\hline Target Range (m) & 2000 & 3000 \\
\hline Target Bearing (deg) & 0 & 0 \\
\hline Initial Target Course (deg) & 135 & 170 \\
\hline Target Course after 300s (deg) & 235 & 295 \\
\hline Target Speed (m/s) & 400 & 400 \\
\hline Target Elevation (deg) & 0 & 0 \\
\hline Observer speed (m/s) & 20 & 20 \\
\hline Observer Course (deg) & 90 & 90 \\
\hline Noise in bearing (1) (deg) & 0.33 & 0.33 \\
\hline Noise in Range (1 $\boldsymbol{\sigma})(\mathbf{m})$ & 10 & 10 \\
\hline Noise in bearing measurements (1 & 0.33 & 0.33 \\
\hline
\end{tabular}

\section{SIMULATION AND RESULTS}

It is assumed that experiment is conducted at favorable environmental conditions. This simulation is carried out on a personal computer using Matlab. The scenario chosen for 
Table. 2. Solution convergence times in seconds

\begin{tabular}{|c|c|c|c|}
\hline \multirow{2}{*}{ Parameters converged } & \multicolumn{2}{c|}{ Scenarios } \\
\cline { 3 - 4 } & Course & 1 & $\mathbf{2}$ \\
\hline \multirow{4}{*}{$\begin{array}{c}\text { Before } \\
\text { Target } \\
\text { maneuver }\end{array}$} & Speed & 54 & 67 \\
\cline { 2 - 4 } & Elevation & 8 & 43 \\
\cline { 2 - 4 } & Total Convergence & 93 & 2 \\
\hline \multirow{3}{*}{$\begin{array}{c}\text { After } \\
\text { Target } \\
\text { maneuver }\end{array}$} & Course & 385 & 460 \\
\cline { 2 - 4 } & Speed & 84 & 43 \\
\cline { 2 - 4 } & Elevation & 8 & 2 \\
\cline { 2 - 4 } & Total Convergence & 385 & 460 \\
\hline
\end{tabular}

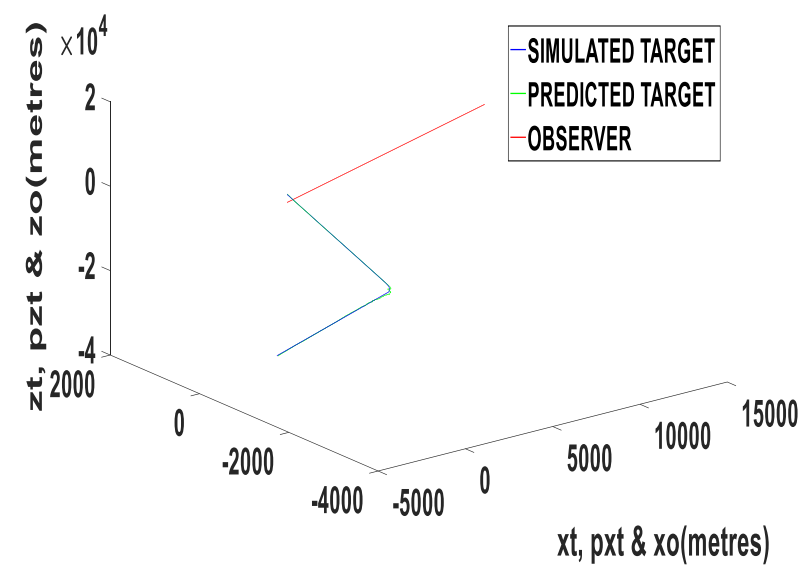

yt, pyt \& yo (metres)

Fig.4 Target and observer coordinates for scenario 2

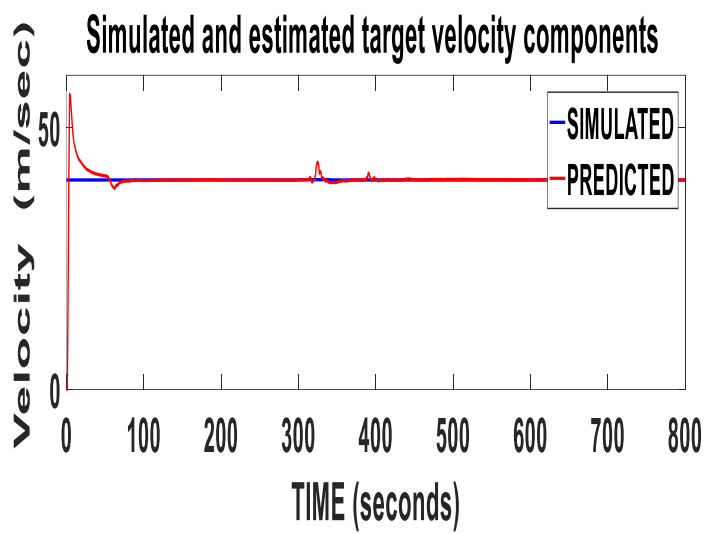

Fig.5True and predicted velocity of target for scenario 2

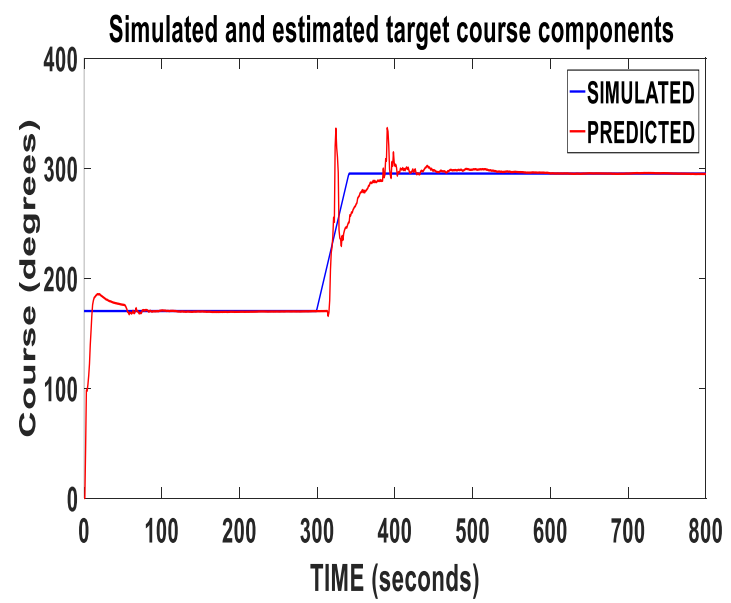

Fig. 6 True and predicted course of target for scenario 2

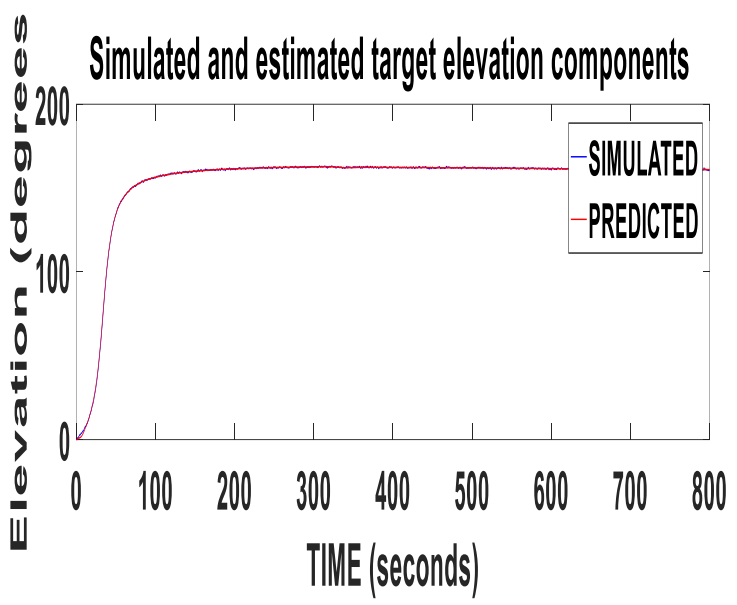

Fig.7 True and predicted elevation of target for scenario 2

\section{CONCLUSION}

Based on the results obtained in simulation, Extended Kalman filter is recommended to estimate target course, speed in active target tracking from UAV systems.

\section{REFERENCES}

1. Edwin E. Westerfield, Dennis J Duven, Larry L.Warnke, "Development of a global positioning system/Sonobuoy system for determining Ballastic missile impact points", John Hopkins APL Technical digest, vol.5, Nov4 1984, pp 335-340

2. Gregory J. Baker and Y.R.M. Bonin, "GPS equippedsonobuoy",http://www.sokkia.com.tw/NOVATEL/Document s/Waypoint/Reports/sonobuoy.pdf

3. S.Koteswara Rao, "Algorithm for detection of maneuvering targets in bearings only passive target tracking”, IEE proc.-sonar Navig., vol.146, No.3, June 1999,pp141-146.

4. S.Koteswara Rao, "Modified gain extended Kalman filter with application to bearings only passive maneuvering target tracking", IEE proc.-Radar Sonar navig., vol 152,No.4,August 2005,pp239-244.

5. M. Kavitha Lakshmi, S. Koteswara Rao, K. Subramanyam, "Passive Object Tracking Using MGEKF Algorithm”, Advances in Intelligent Systems and Computing (Springer Nature Singapore Pte Ltd), vol. 701 , pp. 277-287, 2018.

6. B. Omkar Lakshmi Jagan, S. Koteswara Rao, K. Lakshmi Prasanna, A. Jawahar, Sk. B. Karishma, "Novel estimation algorithm for bearings only target tracking", International journal for engineering and technology, vol.8, No.1. Feb-March 2016, pp238-246.

7. B. Omkar Lakshmi Jagan, S. Koteswara Rao and M. Kavitha Lakshmi, "Concert Assessment of Unscented and Cubature Kalman Filters for Target Tracking", Journal of Advanced Research in Dynamical and Control Systems, Vol. 10, Issue 4, pp.48-56, May 2017.

8. Rao, G.A., Kishore, P.V.V., "Sign language recognition system simulated for video captured with smart phone front camera", International Journal of Electrical and Computer Engineering, Volume 6, Issue 5, 2016, Pages 2176-2187.

9. Madhav, B.T.P., Anilkumar, T., Kotamraju, S.K., "Transparent and conformal wheel-shaped fractal antenna for vehicular communication applications", AEU - International Journal of Electronics and Communications, Volume 91, July 2018, Pages 1-10.

10. Kumar Naik, K., Amala Vijaya Sri, P., "Design of Concentric Circular Ring Patch with DGS for Dual-Band at Satellite Communication and Radar Applications", Wireless Personal Communications, Volume 98, Issue 3, 1 February 2018, Pages 2993-3001.

11. Subhani S.K., Suresh B., Ghali V.S., Empirical mode decomposition approach for defect detection in non-stationary thermal wave imaging ,2016, NDT and E International, Vol: 81, Issue: pp: 39 - 45, ISSN 9638695.

12. Sarath Chandra S., Sastry A.S.C.S.., "Prototype survey of path planning and obstacle avoidance in UAV systems",2018, Lecture Notes in Electrical Engineering ,Vol: 434 ,Issue: ,pp: 555 to:: 563 ,DOI: 10.1007/978-981-10-4280-5_58 ,ISSN: 18761100 9.78981E+12

\section{Published By:}


13. Cheerla S., Ratnam D.V, "RSS based Wi-Fi positioning method using recursive least square (RLS) algorithm", 2018, International Journal of Engineering and Technology (UAE), Vol: 7, Issue: 11, pp: 513 - 517, ISSN: 2227524X.

14. Prasad G.R.K., Babu P.S.S., Khan H., Niak K.K., Generation of radiation characteristics from waveguide slot arrays for satellite tracking radar system,2017 INCEMIC 2015 - 13th International Conference on Electromagnetic Interference and Compatibility, Proceedings, pp:276-278, DOI: 10.1109/INCEMIC.2015.8055894, ISBN: $9.78151 \mathrm{E}+12$

15. Salman M.N., Rao P.T., Rahman M.Z.U., Baseline wander removal in cardiac signals using Variable Step Size Adaptive Noise Cancellers, 2016, Proceedings of the 2016 IEEE International Conference on Wireless Communications, Signal Processing and Networking, Wisp NET 2016, pp: 1529 - 1533

16. Rajendra Prasad C., Bojja P., A review on bio-inspired algorithms for routing and localization of wireless sensor networks,2017 Journal of Advanced Research in Dynamical and Control Systems, Vol:9, issue: Special Issue 18, pp: 1366-1374, ISSN: 1943023X.

17. M. Srinivasan, M. K. Tyagi, K. Radha Rani, M. Suman, B. Loveswara Rao, "An Iterated Extended Risk-Sensitive Filters for Nonlinear Filtering Problems", 2008 IEEE International Conference on Computational Cybernetics, Stara Lesna, Slovakia, 27-29 Nov. 2008.

\section{AUTHORS PROFILE}

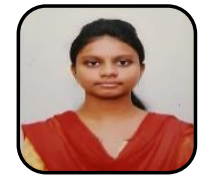

P. Dhatri Shree, studying B. Tech in Electronics and Communication Engineering in Koneru Lakshmaiah Education Foundation, Vaddeswaram, Guntur, AP, India.

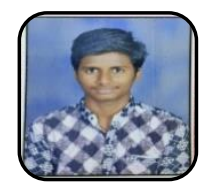

M. Ajay Kumar, studying B. Tech in Electronics and Communication Engineering in Koneru Lakshmaiah Education Foundation, Vaddeswaram, Guntur, AP, India.

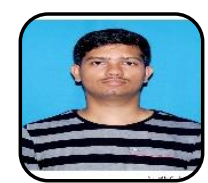

M. Sai Charan,studying B. Tech in Electronics and Communication Engineering in Koneru Lakshmaiah Education Foundation, Vaddeswaram, Guntur, AP, India.

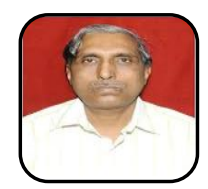

S. Koteswara Rao, former scientist in DRDO, is currently working as Professor in KLEF, Guntur.

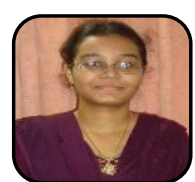

Kausar Jahan, research scholar in KLEF, Guntur, AP. India. 